\title{
FORTBILDUNG
}

Vom Komitee für Prophylaxe und

Therapie Psychischer Erkrankungen P.T.P.

Mitglieder des Komitees: H.-J. Möller, München;

V. Arolt, Münster; M. Berger, Freiburg; M. Gastpar,

Essen; H. Häfner, Mannheim; K. Heinrich, Düsseldorf

C. Kemmerich, Freiburg; M. Linden, Berlin; G. Nissen,

Schwerpunkt

Psychopharmaka in der Hausarztpraxis

27_ Nebenwirkungen:

Die wichtigsten und häufigsten

Würzburg; A. Zacher, Regensburg.

31_ Interaktionen zwischen Psychopharmaka und internistischen Medikamenten

36- Sinnvolle Kombinationen von Psychopharmaka

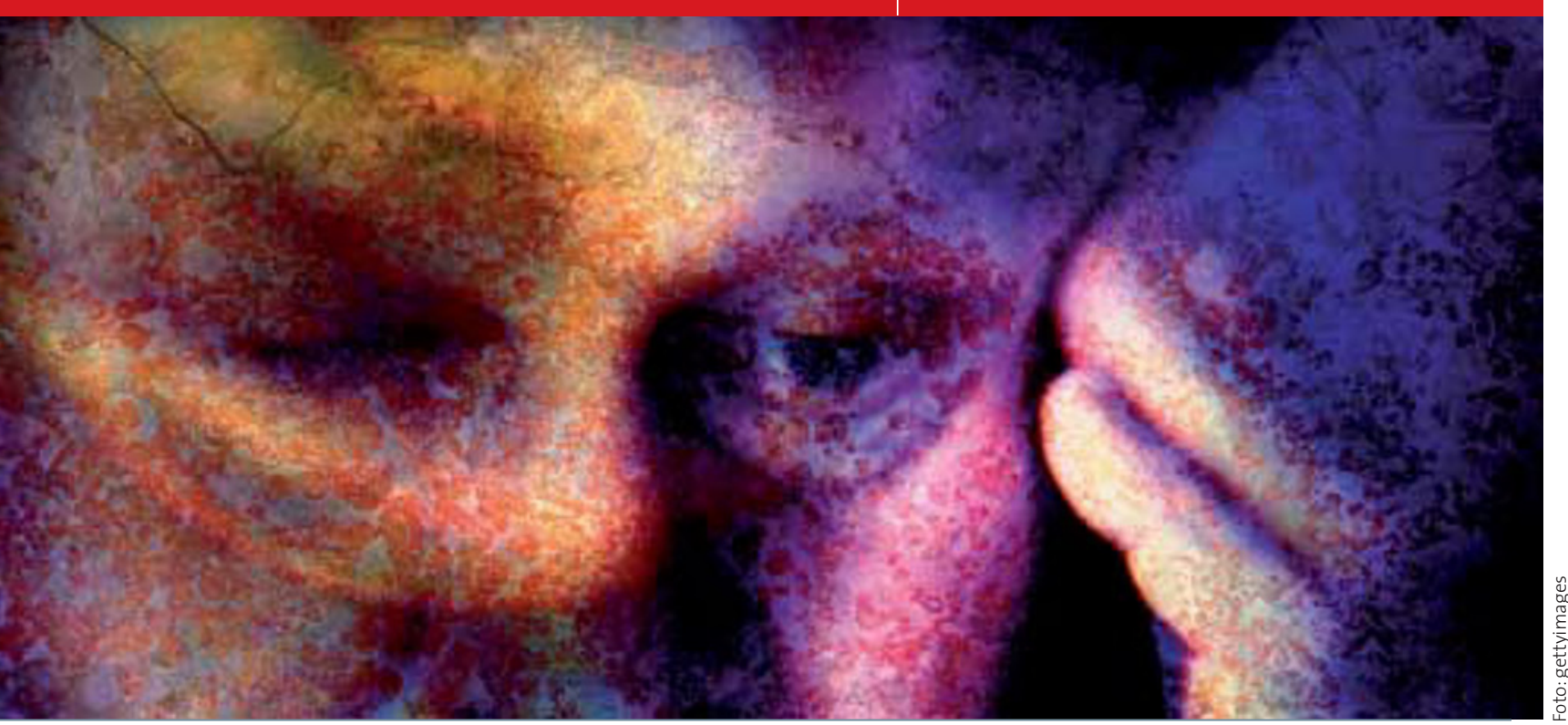

Wenn typische Hausarzt-Patienten Psychopharmaka brauchen

\section{Die wichtigsten Regeln für die Therapie}

- Psychopharmaka nehmen mit über 50 Millionen Verordnungen pro Jahr Platz 3 unter den meistverschriebenen Arzneimittelgruppen ein. Dabei ist in den letzten Jahren eine kontinuierliche Zunahme der Verordnungen von Antidepressiva und Neuroleptika/Antipsychotika zu verzeichnen, während die Zahl der verschriebenen Tranquilizer sinkt.

Insbesondere bei älteren Patienten bestehen neben psychischen Störungen häufig auch körperliche Erkrankungen. Zudem entwickeln 20-25\% der Patienten mit Diabetes mellitus, koronarer Herzkrankheit, Malignomen, nach Herz- oder Hirninfarkt eine behandlungsbedürftige Depression, beiParkinson-Patientennahezu jeder Zweite. So müssen v. a. im höheren Lebensalter wegen der fast immer vorliegenden Multimorbidität Arzneimittelinteraktionen beachtet werden.
Psychische Erkrankungen wie Depressionen verschlechtern den Verlauf vieler körperlicher Erkrankungen. Das Herztodrisiko z. B. ist bei koronarer Herzkrankheit dreifach erhöht, wenn zugleich eine Depression besteht. Deshalb ist neben stützenden ärztlichen Gesprächen der gezielte Einsatz von Psychopharmaka absolut indiziert.

Benzodiazepine und Antidementiva sind hinsichtlich unerwünschter Nebenwirkungen und Arzneimittelwechselwirkungen in der Regel unproblematisch. Beim Einsatz von Antidepressiva und Neuroleptika/Antipsychotika dagegen sind krankheitsbedingte Risiken und Interaktionen mit gleichzeitig verordneten Nicht-Psychopharmaka (z.B. Antihypertensiva, Antikoagulanzien, Antimykotika, Migränemittel, Diuretika) zu beachten. Auch bei dem meist als problemlos

\section{Prof. Dr. med. Dipl.-Psych. Gerd Laux \\ Ärztl. Direktor des Bezirksklinikums Gabersee, \\ Wasserburg am Inn}

angesehenen Phytopharmakon Johanniskraut sowie bei dem zunehmenden Einsatz von Antidepressiva in der chronischen Schmerztherapie sind Interaktionen zu beachten. Darüber hinaus sollte der Hausarzt unbedingt die aktuelle Diskussion über die diabetogene Wirkung bzw. den Zusammenhang zwischen metabolischem Syndrom und neueren atypischen Antipsychotika kennen. Dieser Schwerpunkt soll Ihnen dabei helfen, sich einen Überblick zu verschaffen. 